\title{
Correlation between Magnetic Resonance Imaging and Arthroscopic Findings in Knee Lesions
}

\author{
Octav Russu1,2, Andrei Feier1,2, Emilian Ciorcila1, Georgiana Miler³, Cristian Trambitas², Paul- \\ Gabriel Borodi2 \\ 1 Orthopedics and Traumatology Clinic II, Târgu Mureș, Romania \\ 2 "George Emil Palade" University of Medicine, Pharmacy, Science and Technology, Târgu Mureș, Romania \\ 3 Department of Internal Medicine, Gesundheitszentrum Fricktal, Rheinfelden, Switzerland
}

\section{CORRESPONDENCE}

\section{Andrei Feier}

Str. Gheorghe Marinescu nr. 38

Târgu Mureș, Romania

Tel: +40 747501392

E-mail: andreifeier@gmx.com

\section{ARTICLE HISTORY}

Received: October 22, 2020

Accepted: March 12, 2021
Octav Russu • Str. Mihai Viteazul nr. 31, 540098 Târgu Mureș, Romania. Tel: +40 744266 735, E-mail: octav@ genunchi.ro

Emilian Ciorcila • Str. Mihai Viteazul nr. 31, 540098 Târgu Mures, Romania. Tel: +40 749812 395, E-mail: emilian.ciorcila@yahoo.com

Georgiana Miler • Riburgerstrasse 12, 4310 Rheinfelden, Switzerland. Tel: +41 61835 6666, E-mail: miler_georgiana@yahoo.com

Cristian Trambitas • Str. Gheorghe Marinescu nr. 38, 540142 Târgu Mures, Romania. Tel: +40 745615 113, E-mail: c_trambitas@yahoo.com

Paul Borodi-Gabriel • Jelna nr. 144A, 427017 BistritaNăsăud, Romania. Tel: +40 746349 783, E-mail: borodi.paul@yahoo.com

\begin{abstract}
Background: Magnetic resonance imaging (MRI) is a noninvasive diagnostic method with no known side effects and a high sensitivity for detecting meniscal lesions as well as lesions of the anterior and posterior cruciate ligaments. For a correct and complete diagnosis, MRI results must be interpreted in the context of clinical examination and anamnesis. MRI results can support the surgical indication for arthroscopy, which is a minimally invasive intervention that facilitates the visualization, investigation, diagnosis, and treatment of intra-articular lesions of the knee. The purpose of this study was to assess the correlation between MRI results and the intra-articular lesions detected arthroscopically. Material and methods: This retrospective study evaluated a total of 60 patients admitted and treated between January 1, 2013 and January 1, 2014 in the Clinic of Orthopedics and Traumatology of Mureș County Hospital, Târgu Mureș, Romania. Results: In the 43 patients with lesion of the anterior cruciate ligament, the lesions were seen on MRI in 40 patients (93\%) and confirmed arthroscopically in 37 patients (86\%). In 34 cases (79.1\% of the total of 43), the MRI and arthroscopic results were similar, the Kappa coefficient showing a high degree of correlation (0.90). Conclusion: By assessing the Kappa coefficient to highlight the correlation between MRI results and arthroscopic diagnosis for knee injuries, a statistically relevant correlation between the two methods was found. This suggests that a correct diagnosis can be achieved by both methods.
\end{abstract}

Keywords: knee, intra-articular lesions, magnetic resonance imaging, arthroscopy

\section{INTRODUCTION}

Knee injuries are most commonly encountered as a result of traumas suffered at this level and are frequent because of the increasing popularity of sports such as football, basketball, and skiing in our country. The severity of these lesions depends equally on localization, stretching, physical condition, and muscle strength. Multiple efforts have been made to evaluate the amount of disability 
caused by injuries to knee structures and the success of their treatment by conservative or surgical methods.

Magnetic resonance imaging (MRI) has been successfully used to diagnose knee lesions for more than 20 years and has often been regarded as the noninvasive alternative of diagnostic arthroscopy. Numerous large studies have evaluated the diagnostic capabilities of MRI, with varied outcomes. In day-to-day clinical practice, MRI is routinely used to support the diagnosis of meniscal or ACL injuries prior to recommending arthroscopic examination and surgery. The identification of meniscal tears can be difficult to interpret and depends on both the observer and the sensitivity of the scanner. Similar difficulties may exist in clinical examination as well. The arthroscope provides an easy method to examine the interior of the knee; it provides a good view of the patello-femural joint and is associated with a very low morbidity. The role of arthroscopic examination in the diagnosis of knee problems has been reported many times. Since Burman's description of the use of an arthroscope to visualize joints in a cadaveric model, arthroscopy has risen in popularity to become the most commonly performed orthopedic procedure today. Despite the diverse applications of arthroscopy to examine the shoulder, ankle, wrist, and hip, arthroscopy of the knee has remained the most frequently performed procedure.

Considering that arthroscopy is one of the two major interventions of the last century in orthopedic surgery and MRI is the gold standard paraclinical investigation in knee injuries, the aim of this work was to highlight the correlation between the two methods. For each type of lesion, we aimed to investigate whether they are described both in the operative protocol and the radiological results.

\section{MATERIAL AND METHODS}

A retrospective observational study was conducted during a 12-month period in the Clinic of Orthopedics and Traumatology of Mureș County Hospital, Târgu Mureș, Romania. A total of 60 patients were evaluated. In order to make an objective comparison between the two methods and to reach a conclusion that could be of practical significance, these study assessed lesions of several anatomical structures such as the posterior cruciate ligament (PCL), anterior cruciate ligament (ACL), medial collateral ligament (MCL), medial meniscus, lateral meniscus, persistent vertical septum, reactive synovitis, Baker's cyst, and cartilage lesions.

Patients with the above mentioned knee injuries, aged between 15 and 60 years, who underwent an MRI scan and arthroscopic surgery were included in the study.
TABLE 1. $K$ value

\begin{tabular}{ll}
\hline K value & Degree of agreement \\
\hline$<0.20$ & Slight \\
$0.21-0.40$ & Fair \\
$0.41-0.60$ & Moderate \\
$0.61-0.80$ & Substantial \\
$0.81-1.00$ & Almost perfect \\
\hline
\end{tabular}

Patients with contraindications for MRI (intracerebral aneurysmal clips, cardiac pacemakers, metallic foreign body in the eyes, or middle ear implants), patients with recent knee injury but with no instability in any plane on the clinical examination and negative McMurray test, as well as patients who were unfit for anesthesia were excluded from the study.

A blinded radiologist reported the MRI findings. All arthroscopic procedures were performed in a standard manner by an experienced arthroscopic surgeon, under spinal anesthesia. The diagnostic findings were compared at one level: MRI versus arthroscopic findings.

\section{Statistical analysis}

As a statistical method, we used the Kappa coefficient, which indicates the power of agreement between the two diagnostic methods. Kappa scores take into account the ordering of categorical variables (Table 1). A weighted Kappa score that evaluates agreement between MRI and arthroscopy findings was calculated through a matrix in which MRI ratings were arrayed in the rows and arthroscopy ratings in the columns. The calculation was based on the difference between how much agreement was actually present compared to how much agreement would be expected by chance alone. In our study, the coefficient was calculated using the Statistical Package for Social Sciences (SPSS) for Windows (Armonk, NY: IBM Corp, USA). The Mann-Whitney test was used to analyze demographic data.

The study was conducted in accordance with the principles stipulated in the Declaration of Helsinki. Informed consent was waived by the ethics committee of the Mures County Hospital.

\section{RESULTS}

Baseline demographics and patient characteristics show an average age of $32.8 \pm 11.41$ years (range 15-59 years). Patient gender had an almost equal distribution: $48.3 \%$ (n $=29)$ were women and $51.7 \%(n=31)$ men. The demo- 
graphic data showed no significant differences between the two groups.

Patients included in the study were suffering from knee trauma due to injuries from skiing, football, or falling from the same level, most of them being young patients. Most of the patients (43 out of 60) presented with ACL injuries. Only two patients presented with PCL injuries, diagnosed by both MRI and arthroscopy. As far as meniscus lesions are concerned, most of the subjects (44 out of 60) presented with medial meniscus injuries, and 28 presented with lateral meniscus trauma.

Persistent vertical septum and Baker's cyst were predominantly highlighted by MRI descriptions. Also, it is noteworthy that reactive synovitis was only described in the arthroscopy protocol.

ACL lesions were described on the MRI examination in 40 patients (93\%) and were confirmed arthroscopically in 37 patients $(86 \%)$. In 34 cases $(79.1 \%)$, the MRI and arthroscopic results were similar, the Kappa coefficient showing a high degree of correlation (0.90). Only two patients were identified with a PCL lesion by MRI and arthroscopy. Medial meniscus lesions (anterior and posterior horns) were seen on MRI in 43 patients $(97.7 \%)$ and arthroscopically in 41 patients (93.2\%). In 40 cases (90.9\% from the total of 44) there was an agreement between the two methods regarding the diagnosis, the Kappa coefficient showing a degree of correlation of 0.96 .

Regarding the body of the medial meniscus, the lesion was detected by MRI in nine patients $(81.8 \%)$ and arthroscopically in nine patients (81.8\%). In seven cases (77.8\% from the total of 11 ), there was an agreement between the two methods, the Kappa coefficient showing a correlation of 0.78 , which is considered good.

For the whole medial meniscus (body, anterior and posterior horn), the lesion was detected by MRI in 43 patients (97.7\%) and by arthroscopy in 42 patients (95.5\%). In 41 cases (93.2\% from the total of 44$)$ there was an agreement between the two methods, the Kappa coefficient showing a degree of correlation of 0.96 .

Lateral meniscus injuries (body, anterior and posterior horns) were found by MRI in 23 patients $(82.1 \%$ ) and by arthroscopy in 21 patients $(75 \%)$. In 16 cases $(57.1 \%$ from the total of 28) the diagnosis was concordant between the two methods, the Kappa coefficient of 0.74 indicating a good level of agreement.

In the case of persistent vertical septum, the lesion was detected by MRI in nine patients (69.2\%) and by arthroscopy in 13 patients $(100 \%)$. In nine cases $(69.2 \%$ from the total of 13) the diagnosis was the same for both methods. The Kappa coefficient could not be calculated.
Baker's cyst was highlighted via MRI in six patients (85.7\%) and via arthroscopy in only one patient (14.3\%). This case was also confirmed by MRI. However, the Kappa coefficient of 0.54 indicated a moderate level of agreement.

For cartilage lesions, the injury was found by MRI in 11 patients $(29.7 \%)$, and by arthroscopy in 37 patients. All cases diagnosed by MRI were confirmed by arthroscopy. The Kappa coefficient could not be calculated either for cartilage lesions or for reactive synovitis and lesions of the MCL.

\section{DISCUSSIONS}

Most MRIs were performed in clinics from Târgu Mureș. In order to have more precise data on the sensitivity and specificity of the MRI procedure, a protocol should be established that includes the following information: a detailed patient history, a complete clinical examination, MRI and, if appropriate, an arthroscopic report.

In some situations, the Kappa coefficient of agreement could not be calculated because the number of patients presenting these conditions was insufficient, and the images were depicted on MRI and also arthroscopy. There are numerous studies in recent years addressing this issue, trying to determine which diagnostic method is more accurate. ${ }^{1-23}$ A study on 36 patients evaluated the accuracy of MRI in the diagnosis of cartilage lesions in comparison with arthroscopy. ${ }^{15}$ The results suggested that MRI descriptions can show cartilage damage, but cannot assess the severity of the cartilage injury, the study concluding that arthroscopy remains the most accurate diagnostic method.

Another study, conducted on 185 subjects, compared the diagnostic accuracy of MRI and arthroscopy in ACL and meniscus ruptures. ${ }^{10}$ This study also concluded that arthroscopy was the most accurate diagnostic method, and even a rigorous clinical examination was more reliable than the MRI examination. ${ }^{10}$

Comparing the accuracy of the two methods in diagnosing meniscal lesions, a study conducted on 70 patients found that a clinical examination was at least as suggestive (or even more suggestive in some situations) as MRI images. ${ }^{11}$ The study stressed the importance of clinical examination, which depends on the experience of the surgeon, who in turn relies heavily on the images acquired by arthroscopy. MRI is considered useful when there is a clinical suspicion for a type of lesion but the question arises whether or not to intervene arthroscopically. ${ }^{11-15}$

In another study, the authors aimed to quantify the ability of 3.0 Tesla MRI to detect articular cartilage lesions at the level of the knee. ${ }^{9}$ Joint lesions observed on MRI were grad- 
ed from 1 to 4 and compared with the grades of the Outerbridge classification and those of the International Cartilage Repair Society (ICRS), used in arthroscopic imaging. The articular surface was divided into six regions. However, one limitation consisted in the fact that the MRI assessments were performed by more than one operator, without establishing a coefficient of variance for each lesion. ${ }^{9}$ Based on the MRI images, from a total of 288 joints, 113 (39\%) had a positive diagnosis (from grade 2 to 4 ). The Kappa coefficient applied to the MRI examination, the Outerbridge, and the ICRS classification was $0.13,0.54$, and 0.63 , respectively. When using the ICRS and the Outerbridge classification on MRI, the sensitivity, specificity, and accuracy were $54 \%, 92 \%$, and $75 \%$, respectively. Based on these results, the authors concluded that 3.0 Tesla MRI did not show a significant power in diagnosing cartilage lesions. ${ }^{9}$

\section{CONCLUSIONS}

Out study found a good correlation between MRI and arthroscopy in assessing lesions of the anterior cruciate ligament, medial meniscus, and lateral meniscus. In case of Baker's cyst, there was a moderate diagnostic agreement between the two methods. In the rest of the cases, namely lesions of the posterior cruciate ligament and medial collateral ligament, the existence of reactive synovitis, the persistence of the vertical septum, and cartilage lesions, the Kappa coefficient could not be calculated due to the reduced number of subjects. However, the lesions were mainly diagnosed on MRI, except for reactive synovitis, which was diagnosed only by arthroscopic imaging. In order to assert a correct and complete diagnosis, MRI should be interpreted in the context of a thorough anamnesis and clinical examination. As a final conclusion, there is a strong correlation between the diagnostic power of the compared methods, thus both can be used for a correct diagnosis.

\section{CONFLICT OF INTEREST}

Nothing to declare.

\section{REFERENCES}

1. Bruyère $O$, Burlet $N$, Delmas $P D$, Rizzoli $R$, Cooper $C$, Reginster JY Evaluation of symptomatic slow-acting drugs in osteoarthritis using the GRADE system. BMC Musculoskeletal Disorders. 2008;11:165.
2. Gidwani S, Fairbank A. The orthopaedic approach to managing osteoarthritis of the knee. BMJ. 2004;11:1220-1224.

3. Gross AE. Cartilage resurfacing: filling defects. J Arthrop/asty. 2003:11:1417.

4. Hunziker EB. Articular cartilage repair: basic science and clinical progress. A review of the current status and prospects. Osteoarthritis Cartilage. 2002;11:432-463.

5. Disler DG, McCauley TR, Kelman CG, et al. Fat-suppressed threedimensional spoiled gradient-echo MR imaging of hyaline cartilage defects in the knee: comparison with standard MR imaging and arthroscopy. Am J Roentgenol. 1996;11:127-132.

6. Halbrecht JL, Jackson DW. Office arthroscopy: a diagnostic alternative Arthroscopy. 1992;11:320-326.

7. Mathieu L, Bouchard A, Marchaland JP, et al. Knee MR-arthrography in assessment of meniscal and chondral lesions. Orthop Traumatol Surg Res. 2009:11:40-47.

8. Dzoleva-Tolevska R, Poposka A, Samardziski M, Georgieva D. Comparative analysis of diagnostic methods in meniscal lesions. US National Library of Medicine, Prilozi. 2013;34:79-84;

9. Reed ME, Villacis DC, Hatch GH, Burke WS. 3.0-Tesla MRI and arthroscopy for assessment of knee articular cartilage lesions. Orthopedics. 2013:36:e1060-4

10. Bisson LJ, Kluczynski MA, Hagstrom LS, Marzo JM. A prospective study of the association between bone contusion and intra-articular injuries associated with acute anterior cruciate ligament tear. Am J Sports Med. 2013:41:1801-1807.

11. Siddiqui MA, Ahmad I, Sabir AB. Clinical examination vs. MRI: evaluation of diagnostic accuracy in detecting $\mathrm{ACL}$ and meniscal injuries in comparison to arthroscopy. Pol Orthop Traumatol. 2013:78:59-63.

12. Notohamiprodjo M, Horng A, Kuschel B, Paul D. 3D-imaging of the knee with an optimized 3D-FSE-sequence and a 15-channel knee-coil. Eur J Radiol. 2012;81:3441-3449.

13. Ercin E, Kaya I, Sungur I, Demirbas E. History, clinical findings, magnetic resonance imaging and arthroscopic correlation in meniscal lesions. Knee Surg Sports Traumatol Arthrosc. 2012;20:851-856.

14. Magarelli N, Carducci C, Cannataro G. MR in the evaluation of new anterior cruciate ligament and tibial tunnel position: correlation with clinical and functional features. Radiol Med. 2011;116:1124-1133.

15. von Engelhardt LV, Lahner M, Klussmann A. Arthroscopy vs. MRI for a detailed assessment of cartilage disease in osteoarthritis: diagnostic value of MRI in clinical practice. BMC Musculoskelet Disord. 2010;11:75.

16. Böttcher $\mathrm{P}$, Brühschwein $\mathrm{A}$, Winkels $\mathrm{P}$. Value of low-field magnetic resonance imaging in diagnosing meniscal tears in the canine stifle: a prospective study evaluating sensitivity and specificity in naturally occurring cranial cruciate ligament deficiency with arthroscopy as the gold standard. Vet Surg. 2010;39:296-305.

17. Notohamiprodjo M, Horng A, Pietschmann MF, Müller PE. MRI of the knee at 3T: first clinical results with an isotropic PDfs-weighted 3D-TSEsequence. Invest Radiol. 2009;44:585-597.

18. Esparragoza-Montero R, Rodriguez-Diaz J, Lanier-Dominguez J. Evaluation of meniscal morphology and relation between the diagnostic findings of magnetic resonance imaging and arthroscopy in lesions of the knee. Invest Clin. 2009:50:35-44:

19. Rayan F, Bhonsle S, Shukla DD, Clinical. MRI, and arthroscopic correlation in meniscal and anterior cruciate ligament injuries. Int Orthop. 2009;33:129132

20. Mughetti M, De Masi M, Teodorani A. Computerized tomography false positives in knee medial meniscus injuries. Retrospective study of 400 cases. Radio/ Med. 1998:96:310-312.

21. Brossmann J, Muhle C, Büll CC, Schröder C. Evaluation of patellar tracking in patients with suspected patellar malalignment: cine MR imaging vs arthroscopy. AJR - Am J Roentgenol. 1994:162:361-367;

22. Broderick LS, Turner DA, Renfrew DL. Severity of articular cartilage abnormality in patients with osteoarthritis: evaluation with fast spin-echo MR vs arthroscopy. AJR - Am J Roentgenol. 1994;162:99-103:

23. Tung GA, Davis LM, Wiggins ME. Tears of the anterior cruciate ligament primary and secondary signs at MR imaging. Radiology. 1993;188:661-617. 